\title{
The effect of hospital-acquired infection with Clostridium difficile on length of stay in hospital
}

\author{
Alan J. Forster MD MSc, Monica Taljaard PhD, Natalie Oake MSc, Kumanan Wilson MD MSc, Virginia Roth MD, \\ Carl van Walraven MD MSc
}

See related commentary by Enoch and Aliyu on page 17 and at www.cmaj.ca/lookup/doi/10.1503/cmaj.111449

\section{- ABstract}

Background: The effect of hospital-acquired infection with Clostridium difficile on length of stay in hospital is not yet fully understood. We determined the independent impact of hospital-acquired infection with C. difficile on length of stay in hospital.

Methods: We conducted a retrospective observational cohort study of admissions to hospital between July 1, 2002, and Mar. 31, 2009, at a single academic hospital. We measured the association between infection with hospital-acquired C. difficile and time to discharge from hospital using Kaplan-Meier methods and a Cox multivariable proportional hazards regression model. We controlled for baseline risk of death and accounted for C. difficile as a time-varying effect.

Results: Hospital-acquired infection with C. difficile was identified in 1393 of 136877 admissions to hospital (overall risk $1.02 \%, 95 \%$ confidence interval $[\mathrm{CI}] 0.97 \%-1.06 \%)$. The crude median length of stay in hospital was greater for patients with hospital-acquired C. difficile (34 d) than for those without C. difficile ( $8 \mathrm{~d}$ ). Survival analysis showed that hospital-acquired infection with $C$. difficile increased the median length of stay in hospital by six days. In adjusted analyses, hospitalacquired $C$. difficile was significantly associated with time to discharge, modified by baseline risk of death and time to acquisition of C. difficile. The hazard ratio for discharge by day 7 among patients with hospital-acquired C. difficile was $0.55(95 \% \mathrm{Cl} 0.39-0.70)$ for patients in the lowest decile of baseline risk of death and $0.45(95 \% \mathrm{Cl} 0.32-0.58)$ for those in the highest decile; for discharge by day 28 , the corresponding hazard ratios were $0.74(95 \% \mathrm{Cl}$ $0.60-0.87)$ and $0.61(95 \% \mathrm{Cl} 0.53-0.68)$.

Interpretation: Hospital-acquired infection with $C$. difficile significantly prolonged length of stay in hospital independent of baseline risk of death.

I nfection with Clostridium difficile is associated with poor outcomes for patients. ${ }^{1,2}$ Previous work has determined that, regardless of baseline risk of death, for every 10 patients that acquire $C$. difficile in hospital, 1 patient will die. ${ }^{3}$ Clostridium difficile is also associated with increased health care costs. ${ }^{1,2}$ One of the primary mechanisms by which $C$. difficile increases costs is by increasing the length of time patients spend in hospital. ${ }^{4}$

Previous studies have found that hospitalacquired infection with $C$. difficile increases a patient's length of stay by one to three weeks..$^{2.58}$ However, these estimates are potentially biased. First, previous studies have not accounted for the time-varying nature of this infection. Hospitalacquired infection with $C$. difficile is a variable that is unknown at admission but occurs during the stay in hospital. ${ }^{3,9}$ Treating time-varying variables as

fixed in time-to-event analyses leads to "timedependent bias" and may exaggerate the association between a risk factor and the time to the event of interest. Second, our previous work ${ }^{3}$ has shown that the risk of hospital-acquired infection with C. difficile significantly increases as a patient's baseline risk of death increases; it is, therefore, important to account for risk of death at admission when investigating the association between hospital-acquired $C$. difficile and length of stay.

Because of the importance of an accurate estimate of the impact of $C$. difficile, we conducted a retrospective observational cohort study to determine the independent association between hospital-acquired infection with $C$. difficile and length of stay in hospital. We accounted for each patient's risk of death upon admission and the variable amount of time patients spent in hospital before acquiring $C$. difficile.
Competing interests: Alan Forster, Natalie Oake and Virginia Roth have received peer-reviewed grant funding from the Canadian Patient Safety Institute. Virginia Roth has received grant funding from the Public Health Agency of Canada for the surveillance of Clostridium difficile, and she is employed as an epidemiologist by The Ottawa Hospital. No other competing interests were declared.

This article has been peer reviewed.

Correspondence to: Dr. Alan J. Forster, aforster@ohri.ca

CMAJ 2012. DOI:10.1503 /cmaj.110543 


\section{Methods}

\section{Study design}

This was a retrospective observational study using The Ottawa Hospital Data Warehouse. The Ottawa Hospital is a tertiary-care teaching facility with two acute-care campuses containing 1100 beds. It is the largest adult referral centre for a population of 1.1 million people.

The Data Warehouse contains data from information systems at The Ottawa Hospital, including the patient registration system, the clinical data repository (containing laboratory, pharmacy and radiology information) and the discharge abstract database (containing demographic, diagnostic and procedural information). The study was approved by The Ottawa Hospital Research Ethics Board.

\section{Eligibility criteria}

We included admissions to hospital that occurred after July 1, 2002, for which discharges took place before Mar. 31, 2009. We excluded stays of less than 72 hours, since infection with $C$. difficile deemed to have been acquired in hospital requires a positive test for the organism more than 72 hours after admission. ${ }^{10,11}$ We also excluded admissions of patients younger than 15 years of age and patients admitted to an obstetric service, because the model we used to predict baseline risk in this study was not validated in these populations. ${ }^{12}$ The unit of analysis was the stay in hospital.

We abstracted the following information from the admissions to hospital included in the study: age and sex of patient, dates of admission and discharge, admitting service, comorbidities (for assigning scores on the Charlson comorbidity index) and results of laboratory investigations. We also measured patients' use of the health care system in the year before admission by calculating the number of previous admissions to hospital, visits to the emergency department and total number of days spent as an inpatient at our hospital.

\section{Determination of hospital-acquired C. difficile}

As per guidelines for the surveillance of $C$. diffcile ${ }^{10,11}$ patients were classified as having a hospital-acquired infection if a toxin assay from a sample of liquid stool taken at least 72 hours after admission returned a positive result. Tests with positive results that occurred within two months of a previous positive result were considered a relapse and were not counted.

To determine if $C$. difficile had been acquired in hospital, we applied text-searching techniques to electronic laboratory reports of assays for $C$. difficile toxins to identify positive tests. We then com- pared the date/time of the positive test with the date/time of the patient's admission to determine if the organism was acquired in hospital. We validated the results from this electronic algorithm by comparing them to the results of reports classified manually by infection-control staff; we determined that our algorithm had a sensitivity of $100 \%$.

\section{Outcome}

The primary outcome was time to discharge from hospital. Observation of patient time was censored at death. ${ }^{13}$

\section{Statistical analyses}

We summarized characteristics of included admissions to hospital by $C$. difficile status. We calculated the baseline risk of death for each admission using a logistic regression model ${ }^{12}$ validated in our hospital's population. ${ }^{14}$ This model calculates a patient's risk of dying while in hospital using data available at admission. The model includes age, sex, comorbities, ${ }^{15,16}$ acuity of admission, admitting service and severity of acute disease (summarized using the laboratory-based acute physiology score). ${ }^{12}$

We described times to discharge from hospital by $C$. difficile status using Kaplan-Meier curves that accounted for the time-varying nature of C. difficile. We did so by rearranging our data in the "counting process format." ${ }^{17}$ Data in this format use multiple records per admission to hospital to correspond with a change in status. A patient who acquires $C$. difficile would have two lines of data; the first corresponding to the time between admission and acquisition of $C$. difficile and the second corresponding to the time to discharge or death. Acquisition of $C$. difficile would always be censored in the first observation. Using this format, we tabulated the median times to discharge and the probabilities of remaining in hospital at 7 and 28 days (i.e., the 50th and 90th percentiles of length of stay). We calculated the crude median length of stay by $C$. difficile status. This analysis does not account for the time-varying nature of $C$. difficile.

To calculate the median times to discharge for patients with $C$. difficile, only the days between $C$. difficile acquisition and discharge are attributed to the group with $C$. difficile; the time from admission to $C$. difficile acquisition is attributed to the group that did acquire $C$. difficile in hospital. For admissions to hospital that did not acquire C. difficile, all days between admission and discharge are counted. To control for the effect of baseline risk of death in these analyses, we stratified patients by deciles of risk.

The unadjusted and adjusted associations between hospital-acquired $C$. difficile and time to discharge from hospital were estimated using Cox proportional hazards regression models..$^{18}$ 
Baseline risk of death, hospital-acquired $C$. difficile (specified as a time-varying covariable) ${ }^{19}$ and the interaction between these two variables were the primary predictors in the model. We included the interaction term to account for effect modification by baseline risk of death. The model also included three covariables that influence risk of in-hospital mortality: the number of admissions to hospital in the previous year, the total number of days spent as an inpatient in the previous year and the year of admission to hospital. The number of visits to the emergency department in the previous year was excluded, as it was collinear with the number of admissions to hospital in the previous year. Because we modelled time to discharge, the hazard ratios (HRs) in the models represent the risk of being discharged associated with a particular factor. Thus, an HR of less than 1.00 suggests a decreased risk of discharge, which in turn implies an increased length of stay.

We used fractional polynomial functions to determine the best functional form for baseline risk of death..$^{20-22}$ Covariables were centred about their means to reduce the risk of multicollinearity. ${ }^{23} \mathrm{We}$ accounted for clustering of admissions of the same patient using a marginal approach with robust sandwich estimates for the covarince matrix.. ${ }^{24} \mathrm{To}$ account for nonproportional hazards, we included interaction terms between log-time and hospitalacquired $C$. difficile and between log-time and the fractional polynomial terms for baseline risk of death. We eliminated nonsignificant terms from the model in a stepwise fashion, starting with the highest-order interactions, until all terms remaining in the model were significant at $\alpha=0.05$.

We used the Cox model to generate the timeto-discharge curve that would be expected for patients with hospital-acquired $C$. difficile, had they not acquired the organism. We used the actual covariable values of the patients with C. difficile - changing their hospital-acquired C. difficile statuses to "absent" - to generate the expected time to discharge. The difference between the observed and expected lengths of stay is the increase in length of stay attributed to hospital-acquired $C$. difficile.

\section{Results}

\section{Cohort description}

We included 136877 admissions to hospital in our study (Table 1). About half of the patients admitted to hospital during the study period were female, and the mean age was 63.2 years. Twothirds of patients had at least one chronic disease (as shown by their scores on the Charlson comorbidity index). The median predicted baseline risk of death for all patients was $1.8 \%$ (interquar- tile range [IQR] $0.4 \%-7.6 \%)$. A substantial proportion of patients $(34.6 \%)$ had stayed in our hospital in the year preceding the admission included in our study. In total, 8151 stays in hospital (6.0\%) ended in death.

A total of 1393 patients had hospital-acquired C. difficile (overall risk 1.02\%, 95\% confidence

Table 1: Description of the study cohort

\begin{tabular}{|c|c|c|c|}
\hline \multirow[b]{2}{*}{ Characteristic } & \multirow[b]{2}{*}{$\begin{array}{c}\text { All } \\
(N=136877)\end{array}$} & \multicolumn{2}{|c|}{ Hospital-acquired C. difficile } \\
\hline & & $\begin{array}{c}\text { No } \\
(N=135484)\end{array}$ & $\begin{array}{c}\text { Yes } \\
(N=1393)\end{array}$ \\
\hline Age, mean (SD) & $63.2(17.6)$ & $63.1(17.6)$ & $70.8(15.3)$ \\
\hline Female, \% & 51.6 & 51.5 & 53.8 \\
\hline \multicolumn{4}{|l|}{$\begin{array}{l}\text { Score on Charlson } \\
\text { comorbidity index at } \\
\text { admission, \%* }\end{array}$} \\
\hline 0 & 37.2 & 37.4 & 19.0 \\
\hline $1-2$ & 28.9 & 28.9 & 28.3 \\
\hline $3-4$ & 14.2 & 14.2 & 21.6 \\
\hline $5+$ & 19.7 & 19.5 & 31.1 \\
\hline $\begin{array}{l}\geq 1 \text { admission to hospital } \\
\text { during previous year, } \%\end{array}$ & 34.6 & 34.5 & 44.0 \\
\hline $\begin{array}{l}\geq 1 \text { visit to the } \\
\text { emergency department } \\
\text { during the previous } \\
\text { year, } \%\end{array}$ & 71.8 & 71.7 & 81.4 \\
\hline $\begin{array}{l}\text { Total inpatient time } \\
\text { during previous year, d, } \\
\text { mean (SD) }\end{array}$ & $6.3(16.6)$ & $6.2(16.5)$ & $11.6(24.9)$ \\
\hline $\begin{array}{l}\text { Admitted to a surgical } \\
\text { service, } \%\end{array}$ & 30.7 & 30.8 & 21.4 \\
\hline $\begin{array}{l}\text { Urgent admission to } \\
\text { hospital, } \%\end{array}$ & 74.6 & 74.5 & 82.4 \\
\hline LAPS, $\uparrow$ mean $(S D)$ & $16.2(20.3)$ & $16.1(20.2)$ & $28.7(26.1)$ \\
\hline $\begin{array}{l}\text { Predicted baseline risk } \\
\text { of death, \%, median } \\
\text { (IQR) } ¥\end{array}$ & $1.8(0.4-7.6)$ & $1.8(0.4-7.6)$ & $8.0(2.6-18.4)$ \\
\hline $\begin{array}{l}\text { In-hospital deaths, } \\
\text { no. }(\%)\end{array}$ & $8151(6.0)$ & $7842(5.8)$ & $308(22.1)$ \\
\hline \multicolumn{4}{|c|}{$\begin{array}{l}\text { Note: IQR = interquartile range, LAPS = laboratory-based acute physiology score, SD = } \\
\text { standard deviation. } \\
\text { *The Charlson comorbidity index measures the number and severity of patient comorbidities. } \\
\text { Higher values indicate a greater number of comorbidities. }{ }^{14} \\
\text { tThe LAPS measures the acuity of sickness at hospital admission as reflected by abnormal } \\
\text { results in laboratory tests. Higher values mean greater acute sickness. }{ }^{11} \\
\text { fPredicted baseline risk of death measures a patient's risk of dying while in hospital using } \\
\text { data available at admission. }\end{array}$} \\
\hline
\end{tabular}

Table 2: Timing of hospital-acquired infection with C. difficile Period Time, d, median (IQR)

From admission to detection of $C$. difficile 12 (7-24) From detection of $C$. difficile to discharge 16 (7-33)

Note: $I Q R=$ interquartile range. 
A

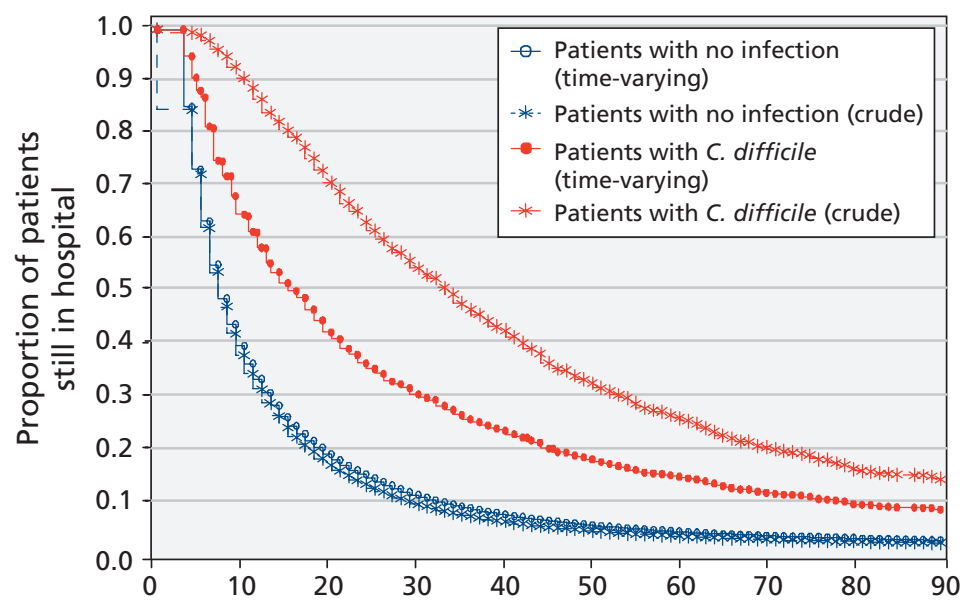

B

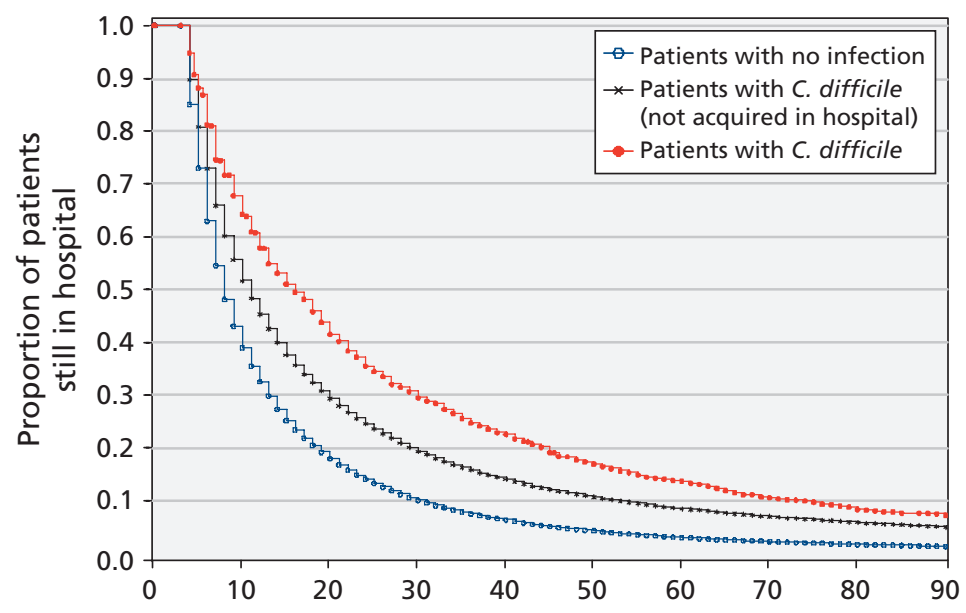

C

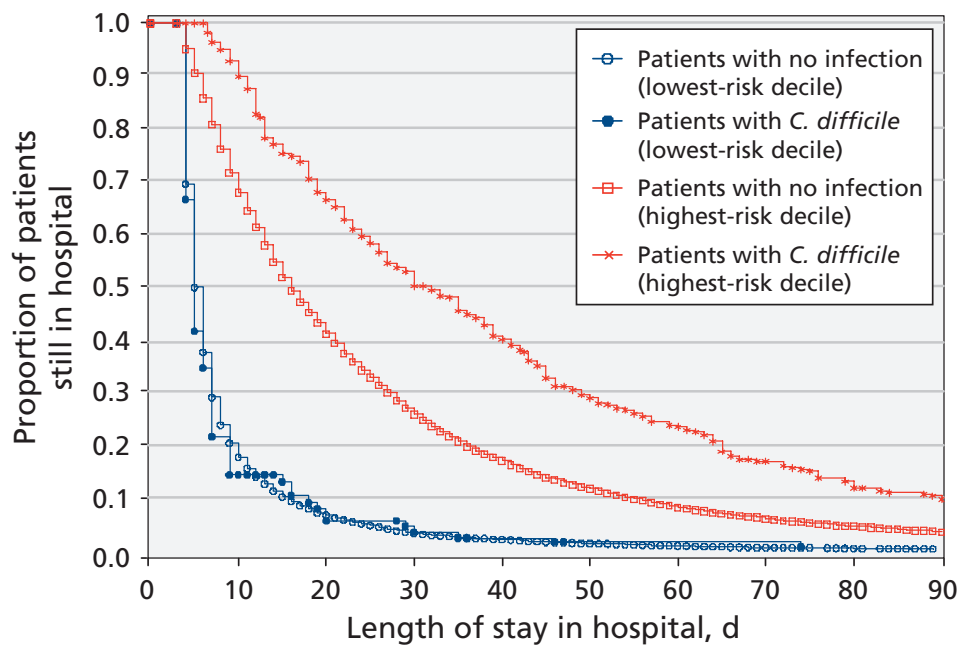

Figure 1: (A) Kaplan-Meier curves for crude and adjusted length of stay in hospital for patients with and without infection with Clostridium difficile. (B) KaplanMeier curves for length of stay in hospital for patients with and without infection with $C$. difficile, accounting for $C$. difficile as a time-varying covariable. (C) Kaplan-Meier curves for length of stay in hospital for patients with and without infection with C. difficile and "low" (first decile) versus "high" (tenth decile) baseline risk of death, accounting for $\mathrm{C}$. difficile as a time-varying covariable. interval $[\mathrm{CI}] 0.97 \%-1.06 \%)$. Compared with the patients who did not have $C$. difficile, patients with $C$. difficile had a higher number of chronic diseases (as shown by higher Charlson scores), higher laboratory-based acute physiology scores and, consequently, a significantly higher baseline risk of death $(8.0 \%$ v. $1.8 \%)$. The proportion of patients who died and who did not have $C$. diffcile was $5.8 \%$, compared with $22.1 \%$ of the patients who had $C$. difficile.

Of hospital-acquired cases of $C$. difficile, $75.5 \%$ were identified on or after the 7 th day spent in hospital. The median number of days from admission to detection of $C$. difficile was 12 (IQR 7-24). For patients with $C$. difficile who were discharged, the median number of days from detection of the organism to discharge was 16 (IQR 7-33) (Table 2).

\section{Time to discharge}

The crude median length of stay was 34 days for patients who had $C$. difficile versus 8 days for those who did not (Figure 1A).

Kaplan-Meier analyses, which accounted for the time-varying nature of $C$. difficile, showed that the median length of stay was longer for patients who had $C$. difficile than for those who did not (16 d v. 8 d) (Appendix 1, available at www .cmaj.ca/lookup/suppl/doi:10.1503/cmaj.110543 /-/DC1, and Figure 1B). As expected, the median lengths of stay for both groups increased with baseline risk of death. In 8 of the 10 deciles for risk of death, the median length of stay was longer for patients with $C$. difficile; differences ranged from 0 days in the lowest-risk decile to 16 days in the highest-risk decile (Figure 1C). Overall, patients with hospital-acquired $C$. difficile were more likely to remain in hospital at 7 and 28 days than those without infection (Appendix 1).

The results of the Cox model (Table 3) show that the log of baseline risk of death, hospitalacquired $C$. difficile and the interaction between these two variables were significant (Table 3). Other variables retained in the model were year of admission, the number of admissions to our hospital in the previous year and the total number of days spent as an inpatient in our hospital in the previous year.

Hospital-acquired $C$. difficile invariably decreased the hazard of discharge (i.e., increased the length of stay in hospital) with increasing baseline risk of death (Figure 2). On day 7, the HR measuring the association between $C$. difficile acquisition and discharge for patients in the lowest decile of baseline risk of death was 0.55 (95\% CI 0.39-0.70), whereas the HR for the highest decile was 0.45 (95\% CI 0.32-0.58); on day 28 , the corresponding HRs were 0.74 
(95\% CI $0.60-0.87)$ and 0.61 (95\% CI 0.53 $0.68)$. Thus, for patients at higher risk at baseline, $C$. difficile had a larger impact on their length of stay in hospital. Furthermore, the ratios increased the longer the patient remained in hospital. Thus, the relative impact of $C$. difficile on the length of stay decreased over time.

The median expected length of stay for patients with $C$. difficile, assuming they had not acquired the infection in hospital, was 10 days (Figure 1B). Since the median time to discharge for patients with hospital-acquired $C$. difficile from Kaplan-Meier analysis was 16 days (Appendix 1), the median increase in length of stay attributed to $C$. difficile was 6 days.

\section{Interpretation}

\section{Main findings}

Hospital-acquired $C$. difficile significantly and independently prolongs the duration of a patient's stay in hospital. The impact of infection with $C$. difficile increases with baseline risk of death and diminishes as the overall length of stay increases. Although a formal cost analysis is beyond the scope of our study, the estimated total cost of caring for patients who acquire this organism in hospital is substantial.

\section{Comparison with other studies}

Previous studies examining the impact of $C$. difficile acquired in hospital on length of stay reported increases of one to three weeks. ${ }^{2,5,6,8,25}$ These are likely overestimates, because $C$. difficile was not treated as a time-varying covariable. Such an approach incorrectly attributes length of stay before acquisition of $C$. difficile to $C$. difficile. To show the impact of not accounting for $C$. difficile as a time-varying covariable, we calculated the difference seen in median length of stay for patients with and without $C$. difficile to be 26 days.

Another notable difference in our analysis compared with those of previous studies is that we accounted for patients' risk of death upon admission to hospital using a validated, highly accurate index that incorporates clinical data. Accurately accounting for baseline risk of death is important, as the patients with the highest risk are more likely to have longer stays in hospital and are thus more likely to acquire $C$. difficile. ${ }^{3}$

Finally, other studies have not evaluated the impact of baseline risk of death and length of stay in hospital on the risk of discharge from hospital. We found that the effect of $C$. difficile acquisition was greater among patients with high baseline risk of death. This suggests that the most intensive efforts to reduce $C$. difficile should occur in units with patients who have the highest risk of death, such as in the intensive care unit and general medical wards. We also found that the relative effect of $C$. difficile acquisition decreased as length of stay increased. Although the relative impact decreases, the absolute effect remains large, given the longer time these patients remain in hospital irrespective of acquiring $C$. difficile.

\section{Limitations}

Our study's main limitation is that the ability to identify the presence and timing of $C$. difficile infections in clinical practice is imperfect. The sensitivity of assays for the $C$. difficile toxin is $55 \%$, and their specificity is $99 \%$. However, there

Table 3: Cox multivariable proportional hazards regression model for variables associated with time to discharge

\begin{tabular}{|c|c|}
\hline Variable & $\mathrm{HR}^{*}(95 \% \mathrm{Cl})$ \\
\hline Log (baseline risk of death) & $0.64(0.63-0.64)$ \\
\hline Log (baseline risk of death) ${ }^{2}$ & $0.97(0.97-0.97)$ \\
\hline Hospital-acquired C. difficile & $0.32(0.25-0.41)$ \\
\hline $\begin{array}{l}\text { Log (baseline risk of death) } \times \text { hospital-acquired } \\
\text { C. difficile }\end{array}$ & $0.96(0.93-0.97)$ \\
\hline Hospital-acquired C. difficile $\times \log$ (time) & $1.24(1.16-1.33)$ \\
\hline Log (baseline risk of death) $\times \log ($ time $)$ & $1.12(1.11-1.12)$ \\
\hline Log (baseline risk of death) $)^{2} \times \log ($ time) & $1.01(1.00-1.01)$ \\
\hline Year of admission & $1.02(1.02-1.02)$ \\
\hline No. of inpatient admissions in the previous year & $1.06(1.05-1.06)$ \\
\hline No. of inpatient days in the previous year & $0.99(0.99-0.99)$ \\
\hline $\begin{array}{l}\text { Note: } \mathrm{Cl}=\text { confidence interval, } \mathrm{HR}=\text { hazard ratio. } \\
{ }^{*} \mathrm{~A} \text { hazard ratio of less than one corresponds to an increas } \\
\text { hazard ratio of greater than one corresponds to a decrease }\end{array}$ & $\begin{array}{l}\text { stay, whereas a } \\
\text { stay. }\end{array}$ \\
\hline
\end{tabular}

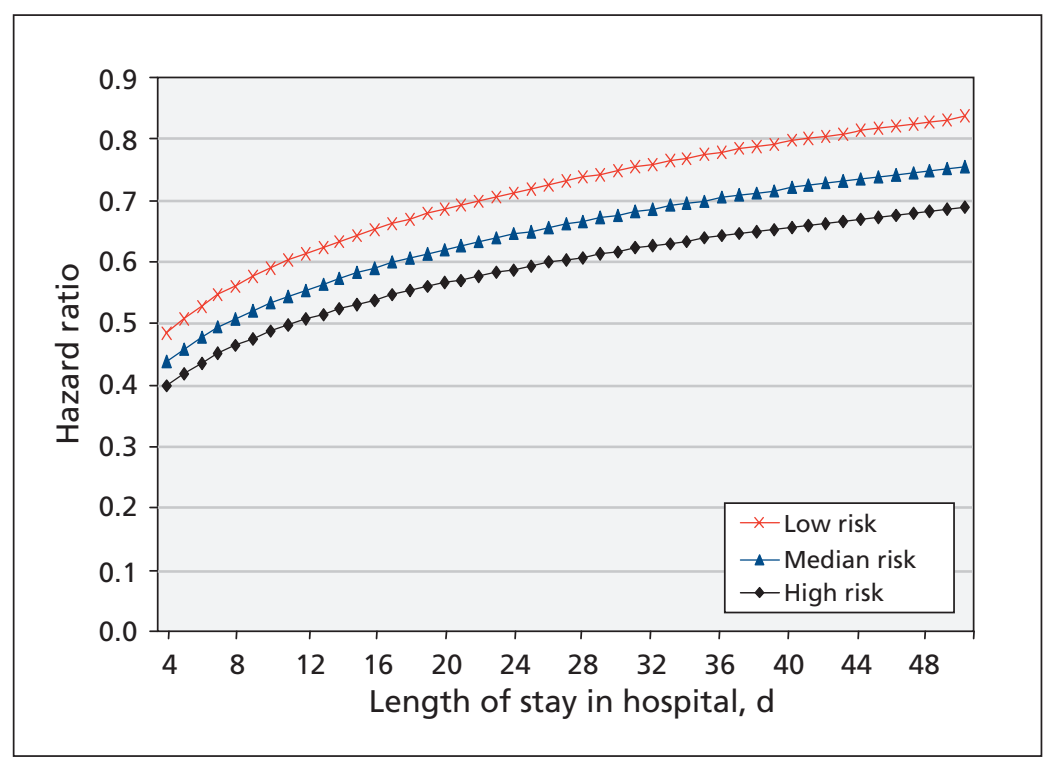

Figure 2: Adjusted hazard ratios for discharge from hospital associated with hospital-acquired infection with Clostridium difficile as a function of time and baseline risk of mortality. Low risk corresponds to the 10th percentile of risk, median risk to the 50th percentile and high risk to the 90th percentile. 
is no accepted gold standard for laboratory diagnosis and toxin testing in the most common method. ${ }^{26}$ In addition, the actual date and time at which $C$. difficile was acquired is likely earlier than the date/time of the positive test result (the date/time used in our study). Therefore, the time from acquisition to discharge for our cohort may be underestimated.

Our choice of primary outcome might be criticized because we censored patients at in-hospital death (i.e., we assumed that censoring is noninformative). We believe the impact of informative censoring to be negligible for two reasons: only $6.0 \%$ of the admissions to hospital were censored owing to death, and our analyses were adjusted for covariables shown to be associated with risk of in-hospital death.

A further limitation was our inability to account for the strain of $C$. difficile with which patients were infected. Our institution does not routinely perform strain analysis on isolates of $C$. difficile.

Finally, the source of data used in this study includes information for our hospital only. We could therefore not study the broader societal impacts of hospital-acquired $C$. difficile.

\section{Conclusion}

We found that the impact of hospital-acquired C. difficile on length of stay is more conservative than previous estimates have suggested. Nevertheless, the impact remains large, especially when one considers the aggregated impact. Future studies should incorporate a formal cost analysis and measure patient outcomes from a broader perspective.

\section{References}

1. Song X, Bartlett JG, Speck KA, et al. Rising economic impact of Clostridium difficile-associated disease in adult hospitalized patient population. Infect Control Hosp Epidemiol 2008;29:823-8.

2. Pépin J, Valiquette L, Cossette B. Mortality attributable to nosocomial Clostridium difficile-associated disease during an epidemic caused by a hypervirulent strain in Quebec. CMAJ 2005; 173:1037-42.

3. Oake N, Taljaard M, van Walraven C, et al. The effect of hospitalacquired Clostridium difficile infection on in-hospital mortality. Arch Intern Med 2010;170:1804-10.

4. Ghantoji SS, Sail K, Lairson DR, et al. Economic healthcare costs of Clostridium difficile infection: a systematic review. $J$ Hosp Infect 2010;74:309-18.

5. Riley TV, Codde JP, Rouse IL. Increased length of hospital stay due to Clostridium difficile associated diarrhoea. Lancet 1995; 345:455-6.

6. Miller MA, Hyland M, Ofner-Agostini M, et al.; Canadian Hospital Epidemiology Committee. Canadian Nosocomial Infection Surveillance Program. Morbidity, mortality, and healthcare burden of nosocomial Clostridium difficile-associated diarrhea in Canadian hospitals. Infect Control Hosp Epidemiol 2002;23:137-40.

7. Wilcox MH, Cunniffe JG, Trundle C, et al. Financial burden of hospital-acquired Clostridium difficile infection. J Hosp Infect 1996;34:23-30

8. Al-Eidan FA, McElnay JC, Scott MG, et al. Clostridium difficileassociated diarrhoea in hospitalized patients. J Clin Pharm Ther 2000;25:101-9.

9. van Walraven C, Davis D, Forster AJ, et al. Time-dependent bias was common in survival analyses published in leading clinica journals. J Clin Epidemiol 2004;57:672-82.

10. Provincial Infectious Diseases Advisory Committee (PIDAC). Best practices document for the management of Clostridum dif- ficile in all health care settings. Toronto (ON): Ontario Ministry of Health and Long-Term Care; 2009.

11. McDonald LC, Coignard B, Dubberke E, et al. Recommendations for surveillance of Clostridium difficile-associated disease. Infect Control Hosp Epidemiol 2007;28:140-5.

12. Escobar GJ, Greene JD, Scheirer P, et al. Risk-adjusting hospital inpatient mortality using automated inpatient, outpatient, and laboratory databases. Med Care 2008;46:232-9.

13. Austin PC, Rothwell D, Tu JV. A comparison of statistical modeling strategies for analyzing length of stay after CABG surgery. Health Serv Outcomes Res Methodol 2002;3:107-33.

14. van Walraven C, Escobar GJ, Greene JD, et al. The Kaiser Permanente inpatient risk adjustment methodology is valid in an external patient population. J Clin Epidemiol 2010;63:798-803.

15. Quan H, Sundararajan V, Halfon P, et al. Coding algorithms for defining comorbidities in ICD-9-CM and ICD-10 administrative data. Med Care 2005;43:1130-9.

16. Schneeweiss S, Wang PS, Avorn J, et al. Improved comorbidity adjustment for predicting mortality in Medicare populations. Health Serv Res 2003;38:1103-20.

17. Ake CF, Carpenter AL. Extending the use of PROC PHREG in survival analysis. Proceedings of the 11th Annual Western Users of SAS Software Conference; 2003 Nov. 5-7; San Francisco (CA). Cary (NC): SAS Institute; 2003.

18. Allison PD. Estimating Cox-regression models with PROC PHREG. Survival analysis using the SAS system. Cary (NC): SAS Institute; 2000. p. 111-84.

19. Fisher LD, Lin DY. Time-dependent covariates in the Cox proportional-hazards regression model. Annu Rev Public Health 1999;20:145-57.

20. Royston P, Altman DG. Regression using fractional polynomials of continuous covariates: parsimonious parametric modelling. Appl Stat 1994;43:429-67.

21. Sauerbrei W, Royston P. Building multivariable prognostic and diagnostic models: transformation of the predictors by using fractional polynomials. J R Stat Soc [Ser A] 1999;162:71-94.

22. Sauerbrei W, Meier-Hirmer C, Benner A, et al. Multivariable regression model building by using fractional polynomials: description of SAS, STATA and R programs. Comput Stat Data Anal 2006;50:3464-85

23. Armitage P, Berry G, Matthews JNS. Modelling continuous data. In: Statistical methods in medical research. Boston (MA): Blackwell Science Limited; 2002. p. 312-77.

24. Gharibvand L, Liu L. Analysis of survival data with clustered events. Proceedings of the SAS Global Forum; 2009 Mar 22-25; Washington (DC). Cary (NC): SAS Institute Inc.; 2009.

25. Vonberg RP, Reichardt C, Behnke M, et al. Costs of nosocomial Clostridium difficile-associated diarrhoea. J Hosp Infect 2008; 70:15-20.

26. Swindells J, Brenwald N, Reading N, et al. Evaluation of diagnostic tests for Clostridium difficile Infection. J Clin Microbiol 2010;48:606-8.

Affiliations: From the Clinical Epidemiology Program (Forster, Taljaard, Oake, Wilson, Roth, van Walraven), Ottawa Hospital Research Institute; the Department of Epidemiology and Community Medicine (Forster, Taljaard, Roth, van Walraven) University of Ottawa; Clinical Quality and Performance Management (Forster, Oake), The Ottawa Hospital; the Department of Medicine, Faculty of Medicine (Forster, Wilson, Roth, van Walraven), University of Ottawa; and the Institute for Clinical Evaluative Sciences (Forster, Wilson, van Walraven), Ottawa, Ont.

Contributors: Alan Forster, Natalie Oake and Carl van Walraven conceived and designed the study protocol and acquired the data. Monica Taljaard, Natalie Oake and Carl van Walraven conducted the analyses. All of the authors were involved in the interpretation of the data, either drafted the article or revised it critically for important intellectual content, and approved the final version submitted for publication.

Funding: The Ottawa Hospital and the Canadian Patient Safety Institute (RFA08-1142-ON) supported this work. The Ottawa Hospital Data Warehouse was funded by the Canadian Foundation for Innovation, the Ontario Innovation Trust and the Ministry of Research and Innovation. Dr. Forster is a career scientist with the Ontario Ministry of Health and Long-Term Care. Dr. Wilson holds a Canada Research Chair in Public Health Policy. 